\title{
A NOTE ON CERTAIN EQUIVALENT NORMS ON TSIRELSON'S SPACE
}

\author{
A. MANOUSSAKIS \\ Department of Sciences, Technical University of Crete, 73100 Chania, Greece \\ E-mail: amanouss@science.tuc.gr
}

(Received 20 June, 2003; accepted 4 December 2003)

\begin{abstract}
We prove that the norm $\|\cdot\|_{n}$ of the space $T\left[\mathcal{S}_{n}, \theta\right]$ and the norm $\|\cdot\|_{n}^{M}$ of its modified version $T_{M}\left[\mathcal{S}_{n}, \theta\right]$ are 3-equivalent. As a consequence, using the results of E. Odell and N. Tomczak-Jaegermann, we obtain that there exists a $K<\infty$ such that for all $n,\|\cdot\|_{n}^{M}$ does not $K$ - distort any subspace of Tsirelson's space $T$.
\end{abstract}

2000 Mathematics Subject Classification. 46B03, 46B20.

1. Introduction. An important and still open question is whether or not there exists a distortable Banach space which is not arbitrarily distortable. The primary candidate for such a space is Tsirelson's space $T$. It is known that for every $1<\lambda<2$, there exists an equivalent norm on $T$ which is a $\lambda$-distortion [11]. Tsirelson's space does not belong to any general class of Banach spaces known to be arbitrarily distortable and in fact recent results show that if there exists a distortable not arbitrarily distortable Banach space $X$, then $X$ must contain a subspace with structure similar to Tsirelson's space. For these and relevant results we refer to the recently published handbooks of Banach spaces [7], [8]. Thus it is of interest to examine all known equivalent norms on $T$ to see if they can arbitrarily distort $T$ or any subspace of $T$. E. Odell and N. Tomczak-Jaegermann, [10], have begun such examination, by considering on $T$ the equivalent norms $\|\cdot\|_{n}$ of the spaces $T_{n}=T\left[\mathcal{S}_{n}, \frac{1}{2^{n}}\right], n \in \mathbb{N}$. They have shown that there exists a constant $K>1$ so that for all $n \in \mathbb{N},\|\cdot\|_{n}$ does not $K$-distort any subspace $Y$ of $T$. Some conditions on the equivalent norms of $T$ which imply that they do not distort $T$, were given earlier in [11]. Our work is a continuation of the work of E. Odell and N. Tomczak-Jaegermann. We consider the modified Tsirelson's space $T_{M}$ and certain equivalent norms of this space. Modified Tsirelson's space $T_{M}$, was introduced by W. B. Johnson [6]. P. Casazza and E. Odell [4], have proved that $T_{M}$ is isomorphic to the original one. Later S. Bellenot [3], gave an elegant argument that shows that $T_{M}$ and $T$ are 2-isomorphic. Our work is inspired by the work of S. Bellenot. We consider the norms $\|\cdot\|_{n}^{M}$ of the spaces $T_{n}^{M}=T_{M}\left[\mathcal{S}_{n}, \frac{1}{2^{n}}\right]$. These norms are equivalent to the norm of $T$ and have much more complexity than the norms of the spaces $T\left[\mathcal{S}_{n}, \frac{1}{2^{n}}\right]$. It is known that for every $n \in \mathbb{N}$ the spaces $T\left[\mathcal{S}_{n}, \frac{1}{2^{n}}\right]$ and $T_{M}\left[\mathcal{S}_{n}, \frac{1}{2^{n}}\right]$ are $2^{n}$-isomorphic. Our main result is that for every $0<\theta<1$ and every $n \in \mathbb{N}$, the spaces $T\left[\mathcal{S}_{n}, \theta\right]$ and $T_{M}\left[\mathcal{S}_{n}, \theta\right]$ are 3 -isomorphic. Combining this result with the work of E. Odell and $\mathrm{N}$. Tomczak-Jaegermann it follows that there exists $K>1$ so that for every $n \in \mathbb{N}$ the norm $\|\cdot\|_{n}^{M}$ does not $K$-distort any subspace of $T$. 
Section 2 contains the relevant terminology and background material. Otherwise our notation is standard as may be found in [9]. More detailed information about Tsirelson's space and some of its variations can be found in [5].

2. Preliminaries. We denote by $c_{00}$ the linear space of finitely supported sequences, and by $\left(e_{i}\right)$ the unit vector basis of $c_{00}$. If $x=\sum_{i} a_{i} e_{i} \in c_{00}$, then $\operatorname{supp} x=$ $\left\{i: a_{i} \neq 0\right\}$. If $E$ is a subset of $\mathbb{N}$, then $E x \in c_{00}$ is defined by $E x=\sum_{i \in E} a_{i} e_{i} . X, Y, \ldots$ will denote separable infinite-dimensional Banach spaces.

A space $(X,\|\cdot\|)$ is arbitrarily distortable, if for all $\lambda>1$, there exists an equivalent norm $|\cdot|$ on $X$ such that

$$
\sup \left\{\frac{|y|}{|z|}: y, z \in S_{Y}\right\}>\lambda \text { for all } Y \subset X .
$$

The norm $|\cdot|$ satisfying (2.1) is said to $\lambda$-distort $X . X$ is $\lambda$-distortable if some norm $\lambda$-distorts $X . X$ is distortable if it is $\lambda$-distortable for some $\lambda>1$.

If $M$ is a subset of $\mathbb{N}$, we denote the set of all finite subsets of $M$ by $[M]^{<}$. For $E, F \subset \mathbb{N}$, we write $E<F$ (respectively $E \leq F$ ), if $\max E<\min F$ (resp. $\max E \leq$ $\min F$ ) or if either one is empty. A subset $\mathcal{F} \subset[\mathbb{N}]^{<}$is hereditary if $G \in \mathcal{F}$ whenever $G \subset F \in \mathcal{F} . \mathcal{F}$ is spreading if whenever $F=\left\{n_{1}, \ldots, n_{k}\right\} \in \mathcal{F}$ with $n_{1}<\cdots<n_{k}$ and $m_{1}<\cdots<m_{k}$ satisfies $n_{i} \leq m_{i}$ for $1 \leq i \leq k$, then $\left\{m_{1}, \ldots, m_{k}\right\} \in \mathcal{F} . \mathcal{F}$ is compact if it is compact in the product topology in $2^{\mathbb{N}}$. A set $\mathcal{F}$ of finite subsets of $\mathbb{N}$ is called regular if it has all three properties. Given $\mathcal{F} \subset[\mathbb{N}]^{<}$, a sequence of finite subsets $\left\{E_{1}, \ldots, E_{n}\right\}$ is said to be $\mathcal{F}$-admissible (resp. $\mathcal{F}$-allowable) if $\left\{\min E_{1}, \ldots, \min E_{n}\right\} \in \mathcal{F}$ and $E_{1}<\cdots<E_{n}$ (respectively $\left\{\min E_{1}, \ldots, \min E_{n}\right\} \in \mathcal{F}$ and $E_{i} \cap E_{j}=\emptyset$ for $i \neq j$ ). Similarly the vectors $x_{i} \in c_{00}, i=1, \ldots, n$ are said to be $\mathcal{F}$-admissible (resp. allowable) if the sequence $\left\{\operatorname{supp} x_{1}, \ldots, \operatorname{supp} x_{n}\right\}$ is $\mathcal{F}$-admissible (resp. allowable).

If $\mathcal{M}$ and $\mathcal{N}$ are regular subsets of $[\mathbb{N}]^{<}$we let

$$
\mathcal{M}[\mathcal{N}]=\left\{\bigcup_{i=1}^{k} F_{i}: F_{i} \in \mathcal{N} \text { for all } i \text { and }\left\{F_{1}, \ldots, F_{k}\right\} \text { is } \mathcal{M} \text {-admissible }\right\},
$$

and

$$
(\mathcal{M})^{3}=\left\{M_{1} \cup M_{2} \cup M_{3}: M_{1}<M_{2}<M_{3} \text { and } M_{1}, M_{2}, M_{3} \in \mathcal{M}\right\} .
$$

The Schreier families $\mathcal{S}_{n}, n \in \mathbb{N}$, were introduced in [1], and are defined as follows: $S_{0}=\{\{n\}: n \in \mathbb{N}\} \cup\{\emptyset\}, \mathcal{S}_{1} \equiv \mathcal{S}=\{F \subset \mathbb{N}: \# F \leq \min F\}$, where $\# F$ denotes the cardinality of $F$. For $n \geq 1, S_{n+1}=\mathcal{S}_{1}\left[S_{n}\right]$. It is easy to see that the Schreier families are regular families of finite subsets of $\mathbb{N}$.

We shall consider also the modified Schreier families $\mathcal{S}_{n}^{M}, n \in \mathbb{N}$, and their relation to the original ones. Their definition and their relation to the original ones are given in the following lemma from [2] whose proof is provided since the relation of $\mathcal{S}_{n}$ and $\mathcal{S}_{n}^{M}$ and especially the relation (2.3) below is an essential tool in our proof.

LEMMA 2.1. [2] For $n<\omega$ define the family $\mathcal{S}_{n}^{M}$ inductively as follows: $\mathcal{S}_{0}^{M}=\mathcal{S}_{0}=$ $\{\{n\}: n \in \mathbb{N}\} \cup\{\emptyset\}, \mathcal{S}_{1}^{M}=\mathcal{S}_{1}=\{F \subset \mathbb{N}: \# F \leq \min F\}$ and for $n \geq 1$,

$$
\mathcal{S}_{n+1}^{M}=\left\{\bigcup_{i=1}^{k} F_{i}: F_{i} \in \mathcal{S}_{n}^{M} \text { for all } i \text { and }\left\{F_{1}, \ldots, F_{k}\right\} \text { is } \mathcal{S} \text {-allowable }\right\} \cup\{\emptyset\} .
$$

Then $S_{n}^{M}=\mathcal{S}_{n}$ for all $n \in \mathbb{N}$. 
Proof. The proof of the lemma is an immediate consequence of the following.

Claim. If $A_{1}, \ldots, A_{k}, k \in \mathbb{N}$, are pairwise disjoint non-empty sets in $\mathcal{S}_{n}$ with $\min A_{1}<\cdots<\min A_{k}$, then there exist non-empty sets $A_{1}^{\prime}<\cdots<A_{k}^{\prime}$ in $\mathcal{S}_{n}$ such that $\cup_{i=1}^{k} A_{i}=\cup_{i=1}^{k} A_{i}^{\prime}$ and $\min A_{i} \leq \min A_{i}^{\prime}$ for $i=1, \ldots, k$.

Proof of the Claim. It is done by induction on $\mathbb{N}$. For $n=0$ it is trivial. Suppose it is true for $n$.

Let $A_{1}, \ldots, A_{k}, k \in \mathbb{N}$, be pairwise disjoint non-empty sets in $\mathcal{S}_{n+1}$ and $\min A_{1}<$ $\ldots<\min A_{k}$. Each $A_{i}$ is of the form $A_{i}=\cup_{j=1}^{m_{i}} B_{j}^{i}$ where $B_{j}^{i} \in \mathcal{S}_{n}$ and, for each $i$, $m_{i} \leq B_{1}^{i}<B_{2}^{i}<\cdots<B_{m_{i}}^{i}$. Let $\left\{B_{j}\right\}_{j=1}^{m_{1}+\cdots+m_{k}}$ be a rearrangement of the family $\left\{B_{j}^{i}\right.$ : $\left.i=1, \ldots, k, j=1, \ldots, m_{i}\right\}$, which satisfies $\min B_{1}<\min B_{2}<\cdots<\min B_{m_{1}+\cdots+m_{k}}$. It is easy to see that, for every $i$,

$$
\min A_{i}=\min B_{1}^{i} \leq \min B_{m_{1}+\ldots+m_{i-1}+1} .
$$

By the inductive assumption, there exists sets $B_{j}^{\prime}, j=1, \ldots, m_{1}+\cdots+m_{k}$, with $B_{j}^{\prime} \in \mathcal{S}_{n}, \cup_{j=1}^{m_{1}+\cdots+m_{k}} B_{j}^{\prime}=\cup_{j=1}^{m_{1}+\cdots+m_{k}} B_{j}$ and such that $B_{1}^{\prime}<B_{2}^{\prime}<\cdots<B_{m_{1}+\cdots+m_{k}}^{\prime}$ and $\min B_{j} \leq \min B_{j}^{\prime}$ for $j=1, \ldots, m_{1}+\cdots+m_{k}$. For $i=1, \ldots, k$, we set

$$
A_{i}^{\prime}=\bigcup_{j=m_{1}+\cdots+m_{i-1}+1}^{m_{1}+\cdots+m_{i}} B_{j}^{\prime}
$$

Then, $A_{1}^{\prime}<A_{2}^{\prime}<\cdots<A_{k}^{\prime}, \cup_{i=1}^{k} A_{i}^{\prime}=\cup_{i=1}^{k} A_{i}$, and for each $i=1, \ldots, k$ we have by (2.2),

$$
m_{i} \leq \min B_{m_{1}+\cdots+m_{i-1}+1} \leq \min B_{m_{1}+\cdots+m_{i-1}+1}^{\prime}
$$

so $A_{i}^{\prime} \in \mathcal{S}_{n+1}$. Moreover, using (2.2) again, we see that

$$
\min A_{i} \leq \min B_{m_{1}+\cdots+m_{i-1}+1}=\min A_{i}^{\prime} .
$$

This completes the proof of the Claim. The lemma follows.

If $\mathcal{F}$ is a regular family of finite subsets of $\mathbb{N}$ and $\theta$ is number with $0<\theta<1$, the Tsirelson space $T[\mathcal{F}, \theta]$ is the completion of $c_{00}$ under the implicit norm

$$
\|x\|=\max \left\{\|x\|_{\infty}, \theta \sup \left\{\sum_{i=1}^{k}\left\|E_{i} x\right\|:\left(E_{i}\right)_{i=1}^{k} \text { is } \mathcal{F} \text {-admissible }\right\}\right\} .
$$

The modified Tsirelson space $T_{M}[\mathcal{F}, \theta]$ is the completion of $c_{00}$ under the implicit norm

$$
\|x\|_{M}=\max \left\{\|x\|_{\infty}, \theta \sup \left\{\sum_{i=1}^{k}\left\|E_{i} x\right\|_{M}:\left(E_{i}\right)_{i=1}^{k} \text { is } \mathcal{F} \text {-allowable }\right\}\right\} .
$$

The classical Tsirelson's space is $T \equiv T\left[\mathcal{S}_{1}, \frac{1}{2}\right]$, and we write $\|\cdot\|$ for the norm of $T$. We also consider the modified Tsirelson's space $T_{M} \equiv T_{M}\left[\mathcal{S}, \frac{1}{2}\right]$, whose norm is denoted by $\|\cdot\|_{M}$. Our main concern are the spaces $T_{n} \equiv T\left[\mathcal{S}_{n}, \frac{1}{2^{n}}\right]$ and their modified 
version $T_{n}^{M} \equiv T_{M}\left[\mathcal{S}_{n}, \frac{1}{2^{n}}\right]$. We shall denote the norms of these spaces by $\|\cdot\|_{n}$ and $\|\cdot\|_{n}^{M}$ respectively. It is clear from the definitions that $\|x\|_{n} \leq\|x\|_{n}^{M}$ for every $x \in c_{00}$.

We shall also make use of the following alternative definition of the norm of $X=T[\mathcal{F}, \theta]$ and $X_{M}=T_{M}[\mathcal{F}, \theta]$, where $\mathcal{F}$ is a regular family of finite subsets of $\mathbb{N}$, and $\theta$ is a number with $0<\theta<1$.

Inductively, we define a subset $K=\cup_{j=0}^{\infty} K_{j}$ of $B_{X^{*}}$ as follows:

$$
K_{0}=\left\{ \pm e_{n}: n \in \mathbb{N}\right\} .
$$

Assume that $K_{j}$ has been defined. We set

$$
\begin{array}{r}
K_{j+1}=K_{j} \cup\left\{\theta\left(f_{1}+\cdots+f_{d}\right): d \in \mathbb{N}, f_{i} \in K_{j}, i=1, \ldots, d,\right. \\
\text { and } \left.\left(f_{i}\right)_{i=1}^{d} \text { is } \mathcal{F} \text {-admissible }\right\} .
\end{array}
$$

Let $K=\cup_{j=0}^{\infty} K_{j}$. Then $K$ is a norming set for $X$ that is, for $x \in X$,

$$
\|x\|=\sup _{f \in K}\langle x, f\rangle \text {. }
$$

The norming set $K^{M}=\cup_{j=0}^{\infty} K_{j}^{M}$ of $X_{M}$ is defined similarly to $K$, namely $K_{0}^{M}=$ $\left\{ \pm e_{n}: n \in \mathbb{N}\right\}$ and for $j \geq 0$,

$$
\begin{array}{r}
K_{j+1}^{M}=K_{j}^{M} \cup\left\{\theta\left(f_{1}+\cdots+f_{d}\right): d \in \mathbb{N}, f_{i} \in K_{j}^{M}, i=1, \ldots, d,\right. \\
\text { and } \left.\left(f_{i}\right)_{i=1}^{d} \text { is } \mathcal{F} \text {-allowable }\right\} .
\end{array}
$$

In what follows, by a tree $\mathcal{T}$ we shall mean a finite set of finite sequences of positive integers, partially ordered by the relation

$$
\alpha \prec \beta \text { iff } \alpha \text { is an initial segment of } \beta,
$$

and with the following property: For every $\alpha \in \mathcal{T},\{\beta: \beta \prec \alpha\} \subseteq \mathcal{T}$. The elements of $\mathcal{T}$ are called nodes. $\mathcal{T}$ has a unique root, the empty sequence which we denote by 0 . The length or level of a sequence $\alpha \in \mathcal{T}$ is denoted by $|\alpha|$. The height of $\mathcal{T}$, denoted by $\mathrm{h}(\mathcal{T})$, is the maximum length of the nodes of $\mathcal{T}$. If $\alpha \in \mathcal{T}$ we denote $S_{\alpha}=$ $\{\beta \in \mathcal{T}: \alpha \prec \beta$ and $|\beta|=|\alpha|+1\}$. The elements of $S_{\alpha}$ are called immediate successors of $\alpha$. If $\alpha \in \mathcal{T}$ and $S_{\alpha}=\emptyset$ we say that $\alpha$ is terminal node. If $\alpha, \beta \in \mathcal{T}$ and $\beta \prec \alpha$ we say that $\beta$ is a predecessor of $\alpha$ or $\alpha$ is a successor of $\beta$.

Let $K$ be the norming set of the space $T[\mathcal{F}, \theta]$ and $K^{M}$ be the norming set of its modified version $T_{M}[\mathcal{F}, \theta]$.

Definition 2.2. Let $\phi \in K$ (resp. $\phi \in K^{M}$ ). An analysis of $\phi$ is a subset $\left\{f_{\alpha}\right\}_{\alpha \in \mathcal{T}}$ of $K$ (resp. $K^{M}$ ), indexed by a tree $\mathcal{T}$ of finite height, such that:

(1) $\phi=f_{0}$

(2) For every $0 \leq s \leq \mathrm{h}(\mathcal{T})$, the elements of the set $\left\{f_{\alpha}: \alpha \in \mathcal{T}\right.$ and $\left.|\alpha|=s\right\}$ are disjointly supported and $\cup_{|\alpha|=s} \operatorname{supp} f_{\alpha} \subset \operatorname{supp} \phi$.

(3) For every $\beta \in \mathcal{T}$, either $f_{\beta}= \pm e_{m_{\beta}}^{*}$ for some $m_{\beta} \in \mathbb{N}$, if $\beta$ is a terminal node of $\mathcal{T}$, or $f_{\beta}=\theta \sum_{\alpha \in S_{\beta}} f_{\alpha}$, and the set $\left\{f_{\alpha}: \alpha \in S_{\beta}\right\}$ is $\mathcal{F}$-admissible (resp. $\mathcal{F}$-allowable).

It is easy to see that every $\phi \in K$ has an analysis. One of the main properties of the norming set is that it is closed under projections on subsets of $\mathbb{N}$, (i.e. if $f \in K$ and $E \subset \mathbb{N}$ then $E f \in K)$. This gives us also that $\left(e_{i}\right)$ is an unconditional basis.

Given a functional $f$ and an analysis $\left(f_{\alpha}\right)_{\alpha \in \mathcal{T}}$ of $f$, we shall adopt the terminology of the tree for the functionals, i.e. we shall call the functionals $f_{\alpha}$ nodes, if $\beta \prec \alpha$ we shall say that $f_{\alpha}$ is successor of $f_{\beta}$ and so on. 
Definition 2.3. Let $f=\left(f_{\alpha}\right)_{\alpha \in \mathcal{T}}$ be a subsequence of $c_{00}$ indexed by a tree $\mathcal{T}$. We say that $f$ or $\mathcal{T}$ is admissible, if for every $\alpha \in \mathcal{T}$ and every $\beta, \gamma \in S_{\alpha}$ with minsupp $f_{\beta}<\operatorname{minsupp} f_{\gamma}$ it holds that $\operatorname{supp} f_{\beta}<\operatorname{supp} f_{\gamma}$.

It is easy to see that if $\mathcal{T}$ is admissible and $\left(\beta_{i}\right)_{i \leq n}$ are the nodes in a level of $\mathcal{T}$ such that (minsupp $f_{\beta_{i}}$ ) $i_{i \leq n}$ is an increasing sequence, we have that $\operatorname{supp} f_{\beta_{i}}<\operatorname{supp} f_{\beta_{i+1}}$ for every $i<n$.

The norms $\|\cdot\|_{n}^{M}$ are equivalent to the norm $\|\cdot\|_{M}$ of $T_{M}$ and it holds that

$$
\|x\|_{n}^{M} \leq\|x\|_{M} \leq 2^{n-1}\|x\|_{n}^{M} .
$$

From (2.3) and the 2-equivalence of the norms $\|\cdot\|$ and $\|\cdot\|_{M},([3,4])$, we get that the norms $\|\cdot\|_{n}^{M}$ are equivalent norms on $T$. Let us explain (2.3).

Let $f$ be a functional in the norming set of $T_{M}\left[\mathcal{S}_{n}, \frac{1}{2^{n}}\right]$ and $\left(f_{\alpha}\right)_{\alpha \in \mathcal{T}}$ be an analysis of $f$. Using the definition of the modified Schreier family $\mathcal{S}_{n}^{M}$, and in particular that $\mathcal{S}_{n}^{M}=\underbrace{\mathcal{S}_{1}^{M}\left[\cdots\left[\mathcal{S}_{1}^{M}\right] \cdots\right]}_{n \text {-times }}$ we define a functional $h$ in the norming set of the space $T_{M}$ and an analysis $\left(h_{\beta}\right)_{\beta \in \mathcal{T}_{1}}$ of $h$, so that for every node $f_{\alpha}, \alpha \in \mathcal{T}$, with $|\alpha|=k$ there exists a node $h_{\beta}, \beta \in \mathcal{T}_{1}$ with $|\beta|=k n$ and $f_{\alpha}=h_{\beta}$. This gives us that the norming set of the space $T_{M}\left[\mathcal{S}_{n}, \frac{1}{2^{n}}\right]$ is contained in the norming set of the space $T_{M}\left[\mathcal{S}, \frac{1}{2}\right]$, and thus the left hand inequality.

In order to get the right hand inequality, for every $f$ in the norming set of the space $T_{M}\left[\mathcal{S}, \frac{1}{2}\right]$ and $\left(f_{\alpha}\right)_{\alpha \in \mathcal{T}}$ an analysis of $f$, we transform its analysis so that the terminal nodes have levels $n, 2 n, 3 n, \ldots$.

More precisely, let $f$ be a functional and $\left(f_{\alpha}\right)_{\alpha \in \mathcal{T}}$ be an analysis of $f$. For $x=$ $\sum a_{i} e_{i} \in c_{00}$ we set $A=\operatorname{supp} x \cap\left\{i: e_{i}^{*}\right.$ is a terminal node of $\left.f\right\}$. Then we have that

$$
f(x)=\sum_{i \in A} \frac{a_{i}}{2^{r_{i}}}
$$

where $r_{i}$ is the level of the terminal node $e_{i}^{*}, i \in A$ (we may assume that $f\left(e_{i}\right) \geq 0$ and $a_{i} \geq 0$ since the basis is unconditional).

If $r_{i} \notin\{n, 2 n, \ldots\}$ we replace $e_{i}^{*}$ by $\frac{1}{2^{l_{i}}} e_{i}^{*}$ where $0<l_{i}<n$ and $r_{i}+l_{i} \in\{n, 2 n, \ldots\}$. This gives us a new tree $\mathcal{T}_{1}$ in which all terminal nodes have levels $n, 2 n, \ldots$ Next, for every node $f_{\alpha}$ with $|\alpha|=0, n, 2 n, \ldots$ we consider as its immediate successors the $\mathcal{S}_{n}$-allowable set, $\left\{f_{\beta}: \alpha \prec \beta, \beta \in \mathcal{T}_{1}\right.$ and $\left.|\beta|=|\alpha|+n\right\}$. This gives us a functional $h$ in the norming set of $T_{M}\left[\mathcal{S}_{n}, \frac{1}{2^{n}}\right]$, which has the same support as $f$. This follows from the replacement of the terminal nodes of $f$. Since every terminal node $e_{i}^{*}$ of $f$ have been moved down $l_{i}$ levels and $0 \leq l_{i} \leq n-1$, we get that

$$
f(x)=\sum_{i \in A} \frac{a_{i}}{2^{r_{i}}} \leq \sum_{i \in A} 2^{l_{i}} \frac{a_{i}}{2^{r_{i}+l_{i}}} \leq 2^{n-1} h(x) \leq 2^{n-1}\|x\|_{n}^{M} .
$$

From (2.4) it is clear that if we change the position of a terminal node in the tree without changing its level, the estimation remains the same. This will be the main ingredient in the proof for the equivalence of the norms $\|\cdot\|_{n}$ and $\|\cdot\|_{n}^{M}$.

3. The spaces $T_{n}$ and $T_{n}^{M}$ are 3-isomorphic. We consider the regular families $\mathcal{A}_{n}=\{F \subset \mathbb{N}: \# F \leq n\}, n \in \mathbb{N}$, of finite subsets of $\mathbb{N}$. Let $\mathcal{F}_{n}=\mathcal{S}_{n}\left[\mathcal{A}_{2}\right]$. First we shall consider the auxiliary space $Y=T\left[\mathcal{S}_{n}\left[\mathcal{A}_{2}\right], \frac{1}{2^{n}}\right]$. We prove in Proposition 3.2, that 
the spaces $T_{n}$ and $Y$ are 3-isomorphic. Next we show, Proposition 3.4, that given a functional $f$ in the norming set of the space $T_{n}^{M}$, we can produce a functional $g$ in the norming set of the space $Y$, that dominates the estimation of $f$ for every $x \in c_{00}$. This will give us that the norm of the space $T_{n}^{M}$ is dominated by the norm of the space $Y$. From the 3-equivalence of the norms of the spaces $Y$ and $T_{n}$ we get that the spaces $T_{n}$ and $T_{n}^{M}$ are 3-isomorphic.

We begin with an auxiliary lemma.

Lemma 3.1. Let $n \in \mathbb{N}$. Then it holds that $\mathcal{F}_{n}\left[\mathcal{A}_{3}\right]=\left[\mathcal{S}_{n}\left[\mathcal{A}_{2}\right]\right]\left[\mathcal{A}_{3}\right] \subset\left(\mathcal{S}_{n}\right)^{3}$.

Proof. We shall prove the result by induction on $n \in \mathbb{N}$. For $n=1$,

$$
\left[\mathcal{S}_{1}\left[\mathcal{A}_{2}\right]\right]\left[\mathcal{A}_{3}\right]=\mathcal{S}_{1}\left[\mathcal{A}_{2}\left[\mathcal{A}_{3}\right]\right]=\mathcal{S}_{1}\left[\mathcal{A}_{6}\right]
$$

Let $k \leq m_{1}<\cdots<m_{k}$ and

$$
m_{i} \leq \lambda_{(i-1) 6+1}<\lambda_{(i-1) 6+2}<\lambda_{(i-1) 6+3}<\lambda_{(i-1) 6+4}<\lambda_{(i-1) 6+5}<\lambda_{6 i}<m_{i+1}
$$

for every $i=1, \ldots, k$. We set $G=\left\{\lambda_{1}, \ldots, \lambda_{6 k}\right\}$. From the assumptions we have that $k \leq G$. We consider the sets

$$
\begin{gathered}
F_{1}=\{k, k+1, \ldots, 2 k-1\}, \quad F_{2}=\{2 k, 2 k+1, \ldots, 4 k-1\}, \\
F_{3}=\{4 k, 4 k+1, \ldots, 7 k-1\} .
\end{gathered}
$$

Each of the sets $F_{i}, i=1,2,3$ belongs to the family $\mathcal{S}$ since $\# F_{i} \leq \min F_{i}$. We may write $G=\cup_{i=1}^{3} G_{i}$ where $G_{i}=\left\{\lambda_{j+1-k}: j \in F_{i}\right\}$ for $i=1,2,3$. Then $G_{i}$ is a spreading of $F_{i}$ and hence belongs to the Schreier family $\mathcal{S}$. Thus $G \in\left(\mathcal{S}_{1}\right)^{3}$. that

Assume that the result holds for $n$, i.e. $\mathcal{F}_{n}\left[\mathcal{A}_{3}\right]=\left[\mathcal{S}_{n}\left[\mathcal{A}_{2}\right]\right]\left[\mathcal{A}_{3}\right] \subset\left(S_{n}\right)^{3}$. Then we have

$$
\mathcal{F}_{n+1}\left[\mathcal{A}_{3}\right]=\left[\mathcal{S}_{n+1}\left[\mathcal{A}_{2}\right]\right]\left[\mathcal{A}_{3}\right]=\mathcal{S}_{1}\left[\mathcal{S}_{n}\left[\mathcal{A}_{2}\right]\right]\left[\mathcal{A}_{3}\right]=\mathcal{S}_{1}\left[\mathcal{F}_{n}\left[\mathcal{A}_{3}\right]\right] \subset \mathcal{S}_{1}\left[\left(\mathcal{S}_{n}\right)^{3}\right] \subset\left(\mathcal{S}_{n+1}\right)^{3}
$$

The last inclusion relation, i.e. $\mathcal{S}_{1}\left[\left(\mathcal{S}_{n}\right)^{3}\right] \subset\left(\mathcal{S}_{n+1}\right)^{3}$, follows immediately from the associativity of the operation $\mathcal{M}[\mathcal{N}]$, and the fact that $(\mathcal{M})^{3}=\mathcal{A}_{3}[\mathcal{M}]$.

PROPOSITION 3.2. For every $x \in c_{00}$ we have the inequality

$$
\|x\|_{n} \leq\|x\|_{Y} \leq 3\|x\|_{n} .
$$

Proof. An easy inductive argument, using that $S_{n} \subset \mathcal{S}_{n}\left[\mathcal{A}_{2}\right]$, gives us that the norming set of $T_{n}=T\left[\mathcal{S}_{n}, \frac{1}{2^{n}}\right]$ is contained in the norming set of $Y=T\left[\mathcal{S}_{n}\left[\mathcal{A}_{2}\right], \frac{1}{2^{n}}\right]$, and hence the left hand inequality.

For the right hand inequality we shall prove that for every functional $f$ in the norming set $K=\cup_{j=0}^{\infty} K_{j}^{Y}$ of the space $Y$ there exist three functionals $g_{1}, g_{2}, g_{3}$ in the norming set of the space $T_{n}=T\left[\mathcal{S}_{n}, \frac{1}{2^{n}}\right]$ so that

$$
f(x) \leq g_{1}(x)+g_{2}(x)+g_{3}(x), \bigcup_{i=1}^{3} \operatorname{supp} g_{i} \subset \operatorname{supp} f \text { and } g_{1}<g_{2}<g_{3} .
$$

For $f=e_{k}^{*} \in K_{0}^{Y}$ (3.1) trivially holds. Assume that the result holds for every $f \in K_{j}^{Y}$, and let $f=\frac{1}{2^{n}} \sum_{i \in F} f_{i} \in K_{j+1}^{Y}$. Then we have that $\left\{\right.$ minsupp $\left.f_{i}: i \in F\right\} \in \mathcal{S}_{n}\left[\mathcal{A}_{2}\right]$ and the functionals $\left(f_{i}\right)_{i \in F} \subset K_{j}^{Y}$ have successive support. 
By the inductive hypothesis for every $i \in F$ there exist three functionals $g_{i}^{1}, g_{i}^{2}, g_{i}^{3}$ in the norming set of the space $T\left[\mathcal{S}_{n}, \frac{1}{2^{n}}\right]$ so that (3.1) holds for $f_{i}$ and $g_{i}^{1}, g_{i}^{2}, g_{i}^{3}$ i.e.

$$
f_{i}(x) \leq g_{i}^{1}(x)+g_{i}^{2}(x)+g_{i}^{3}(x), \bigcup_{j=1}^{3} \operatorname{supp} g_{i}^{j} \subset \operatorname{supp} f_{i} \text { and } g_{i}^{1}<g_{i}^{2}<g_{i}^{3} .
$$

We have that $\left\{\right.$ minsupp $\left.f_{i}: i \in F\right\} \in \mathcal{S}_{n}\left[\mathcal{A}_{2}\right]$ and therefore

$$
\left\{\text { minsupp } g_{i}^{1} \text {, minsupp } g_{i}^{2} \text {, minsupp } g_{i}^{3}: i \in F\right\} \in\left[\mathcal{S}_{n}\left[\mathcal{A}_{2}\right]\right]\left[\mathcal{A}_{3}\right] \text {. }
$$

From Lemma 3.1 we have that $\left\{\right.$ minsupp $g_{i}^{1}$, minsupp $g_{i}^{2}$, minsupp $\left.g_{i}^{3}: i \in F\right\} \in\left(\mathcal{S}_{n}\right)^{3}$. Therefore the functional $\frac{1}{2^{n}} \sum_{i \in F}\left(g_{i}^{1}+g_{i}^{2}+g_{i}^{3}\right)$ can be written as the sum of the three successive functionals $g_{k}=\frac{1}{2^{n}} \sum_{A_{k}} g_{i}^{j}, k=1,2,3$, where $A_{1}<A_{2}<A_{3}$ are such that $A_{1} \cup A_{2} \cup A_{3}=\left\{\right.$ minsupp $g_{i}^{1}$, minsupp $g_{i}^{2}$, minsupp $\left.g_{i}^{3}: i \in F\right\} \in\left(\mathcal{S}_{n}\right)^{3}$. It follows from (3.2) that

$$
\begin{gathered}
f(x)=\frac{1}{2^{n}} \sum_{i \in F} f_{i}(x) \leq \frac{1}{2^{n}} \sum_{i \in F}\left(g_{i}^{1}+g_{i}^{2}+g_{i}^{3}\right)(x)=\left(g_{1}+g_{2}+g_{3}\right)(x), \\
\bigcup_{i=1}^{3} \operatorname{supp} g_{i} \subset \operatorname{supp} f \text { and } g_{1}<g_{2}<g_{3} .
\end{gathered}
$$

Therefore (3.1) holds for $f$ and $g_{1}, g_{2}, g_{3}$. This completes the proof.

REMARK 3.3. Let us observe that Proposition 3.2 is independent of the number $\frac{1}{2^{n}}$, that is, it holds also for the norms of the spaces $T\left[\mathcal{S}_{n}, \theta\right]$ and $T\left[\mathcal{S}_{n}\left[\mathcal{A}_{2}\right], \theta\right]$, for every $0<\theta<1$.

Let $f$ be a functional in the norming set of the space $T_{M}\left[\mathcal{S}_{n}, \frac{1}{2^{n}}\right]$ and $\left(f_{\alpha}\right)_{\alpha \in \mathcal{T}}$ be an analysis of $f$. In the next proposition, following the analysis of $f$, we shall construct a functional $g$ in the norming set of the space $Y=T\left[\mathcal{S}_{n}\left[\mathcal{A}_{2}\right], \frac{1}{2^{n}}\right]$ which gives the same estimation as $f$. As a consequence we get that $\|x\|_{n}^{M} \leq\|x\|_{Y}$, for every $x \in c_{00}$. Our proof is based on the properties of the tree, and the basic tool in the proof is the notion of the good terminal node, Definition 3.5. The definition of a good terminal and Lemma 3.6, with slight modifications, have been taken from [3], Theorem 2.1, where the terminology good leaf is used instead. Our proof is a descendant of the proof in [3].

Proposition 3.4. Let $f$ be a functional in the norming set of the space $T_{M}\left[\mathcal{S}_{n}, \frac{1}{2^{n}}\right]$ and $\left(f_{\alpha}\right)_{\alpha \in \mathcal{T}}$ be an analysis of $f$. Then there exists a functional $g$ in the norming set of the space $Y=T\left[\mathcal{S}_{n}\left[\mathcal{A}_{2}\right], \frac{1}{2^{n}}\right]$ and an analysis $\left(g_{a}\right)_{\alpha \in \mathcal{T}_{1}}$ of $g$, so that

the trees $\mathcal{T}$ and $\mathcal{T}_{1}$ have the same terminal nodes, and the level of each terminal node is the same in both trees $\mathcal{T}$ and $\mathcal{T}_{1}$.

It follows that $\|x\|_{n}^{M} \leq\|x\|_{Y}$ for every $x \in c_{00}$.

Proof. It is clear from (3.3) that the functional $g$ has the same support as $f$ and gives the same estimation. This gives us also that $\|x\|_{n}^{M} \leq\|x\|_{Y}$ for every $x \in c_{00}$..

We shall transform $\mathcal{T}$ in several steps in order to get the functional $g$. Actually in every step we construct a part of its analysis, starting from the level $h(\mathcal{T})$. 
Claim 1. We may transform the analysis $\left(f_{\alpha}\right)_{\alpha \in \mathcal{T}}$ to satisfy the following:

If $\alpha, \beta$ are nodes on the same level with minsupp $f_{\alpha}<\operatorname{minsupp} f_{\beta}$ and

$$
\gamma \in S_{\alpha}, \delta \in S_{\beta} \text { then minsupp } f_{\gamma}<\operatorname{minsupp} f_{\delta} \text {. }
$$

In particular the nodes in every level of $\mathcal{T}$ are ordered following the natural order of the minimum of their support.

Proof of Claim 1. To achieve this property first note that the nodes in level $j$ form an $\mathcal{S}_{n j}$-allowable set, and therefore $\left\{\right.$ minsupp $\left.f_{\alpha}:|\alpha|=j\right\} \in \mathcal{S}_{n j}^{M}$. From Lemma 2.1 we have $S_{k}^{M}=S_{k}$ for every $k \in \mathbb{N}$, and especially the Claim holds. Working from the level $\mathrm{h}(\mathcal{T})$ and moving toward the root we rearrange in every step the (new) nodes of every level $j$, so that $\left\{\operatorname{minsupp} f_{\alpha}:|\alpha|=j\right\} \in \mathcal{S}_{n j}$. This gives us also that the nodes in every level of $\mathcal{T}$ are ordered following the natural order of the minimum of their support.

For any such transformation we obtain new nodes which are also denoted by $f_{\alpha}$. Lemma 2.1 and the Claim ensure that the new nodes remain functionals in the norming set of the space $T_{M}\left[\mathcal{S}_{n}, \frac{1}{2^{n}}\right]$.

Note also that if we have arranged the nodes in the $j$-level to be an $\mathcal{S}_{n j}$-admissible set, any such transformation in the $(j-1)$-level does not have any affect in the support of nodes in the $j-$ level.

Indeed, in the level $h(\mathcal{T})$ we just rearrange the terminal nodes in successive order with respect to their support, while in any level $<\mathrm{h}(\mathcal{T})$ any such transformation takes place either between terminal nodes or between terminal nodes and nodes which have successors.

No such operation changes the estimation of the functional, since the terminal nodes do not change level and hence the new functional gives the same estimation.

After we complete these transformations, it is easy to see that the nodes which prevent $\left(f_{\alpha}\right)_{\alpha \in \mathcal{T}}$ to be an admissible functional are the terminal nodes. To proceed we give the following definitions.

Definition 3.5. A node $f_{\beta}$ is to the lower left of node $f_{\alpha}$, if $|\beta| \geq|\alpha|$, and if $\gamma$ is $\beta^{\prime}$ s unique predecessor in the level of $\alpha$, then minsupp $f_{\gamma}<\operatorname{minsupp} f_{\alpha}$. A terminal node $f_{\alpha}= \pm e_{m_{\alpha}}^{*}$ is said to be a good terminal node if for all nodes $f_{\beta}$ to the lower left of $f_{\alpha}$, we have that minsupp $f_{\beta}<\operatorname{minsupp} f_{\alpha}$. If the terminal node is not good, we will say $f_{\alpha}$ is bad.

In the sequel when we consider terminal nodes $f_{\alpha}= \pm e_{m_{\alpha}}^{*}$ and examine the property of being good or bad terminal node, in many cases we shall omit the sign of $e_{m_{\alpha}}^{*}$ since this property is independent of the sign.

LEMMA 3.6. If $\mathcal{T}$ has only good terminal nodes, then $\mathcal{T}$ is admissible.

Proof of Lemma. Let $\alpha$ be a node in $\mathcal{T}$ and $\beta, \gamma \in S_{\alpha}$ so that minsupp $f_{\beta}<$ minsupp $f_{\gamma}$. Let $\lambda_{\beta}=$ maxsupp $f_{\beta}$ and $m_{\gamma}=\operatorname{minsupp} f_{\gamma}$. It follows that $e_{\lambda_{\beta}}^{*}$ and $e_{m_{\gamma}}^{*}$ are terminal nodes. We need to show that $e_{\lambda_{\beta}}^{*}<e_{m_{\gamma}}^{*}$ which is equivalent to maxsupp $f_{\beta}<$ minsupp $f_{\gamma}$. If $\left|e_{\lambda_{\beta}}^{*}\right| \geq\left|e_{m_{\gamma}}^{*}\right|$ it follows from (3.4) that $e_{\lambda_{\beta}}^{*}$ is to the lower left of $e_{m_{\gamma}}^{*}$.

Indeed, let $\left\{f_{\beta}=f_{\beta_{1}} \prec f_{\beta_{2}} \prec \cdots \prec f_{\beta_{m}}=e_{\lambda_{\beta}}^{*}\right\}$ be the predecessors of $e_{\lambda_{\beta}}^{*}$ until the level of $f_{\beta}$, and $\left\{f_{\gamma}=f_{\gamma_{1}} \prec f_{\gamma_{2}} \prec \cdots \prec f_{\gamma_{k}}=e_{m_{\gamma}}^{*}\right\}$ be the predecessors of $e_{m_{\gamma}}^{*}$ until the level of $f_{\gamma}$. Since $\left|e_{\lambda_{\beta}}^{*}\right| \geq\left|e_{m_{\gamma}}^{*}\right|$, it follows that $k \leq m$. Inductively, from (3.4), we get that 
minsupp $f_{\beta_{i}}<\operatorname{minsupp} f_{\gamma_{i}}$ for every $i \leq k$. Since $e_{\lambda_{\beta}}^{*}$ is a successor of $f_{\beta_{k}}$ we get that $e_{\lambda_{\beta}}^{*}$ is to the lower left of $e_{m_{\gamma}}^{*}$ and therefore $e_{\lambda_{\beta}}^{*}<e_{m_{\gamma}}^{*}$, since $e_{m_{\gamma}}^{*}$ is a good terminal.

If $\left|e_{\lambda_{\beta}}^{*}\right|<\left|e_{m_{\gamma}}^{*}\right|$, let $f_{\delta}$ be the predecessor node of $e_{m_{\gamma}}^{*}$ in the level of $e_{\lambda_{\beta}}^{*}$. Let $m_{\delta}=\operatorname{minsupp} f_{\delta}$. From the definition of the analysis it holds that $e_{m_{\delta}}^{*} \leq e_{m_{\gamma}}^{*}$, and from property (3.4), as in the previous case, it follows easily that $e_{\lambda_{\beta}}^{*}<e_{m_{\delta}}^{*}$ and hence $e_{\lambda_{\beta}}^{*}<e_{m_{\gamma}}^{*}$.

Our aim is to transform $\mathcal{T}$ in such a way that it contains only good terminal nodes, but losing the admissibility in the way that every node to have at most $\mathcal{S}_{n}\left[\mathcal{A}_{2}\right]$-admissible immediate successors, and obtaining the same estimation.

INDUCTIVE HYPOTHESIS. Assume that we have transformed $\mathcal{T}$ in such way so that for the levels $\geq \mathrm{h}(\mathcal{T})-i, i \geq 0$, the following holds:

(1) The terminal nodes in the levels $\geq \mathrm{h}(\mathcal{T})-i$ are good terminal nodes.

(2) Every node $f_{\alpha}$ in the levels $\geq \overline{\mathrm{h}}(\mathcal{T})-i$ belongs to the norming set of the space $Y$.

(3) For every node $f_{\alpha}$ in the levels $\geq \mathrm{h}(\mathcal{T})-i-1$, the set $\left\{f_{\beta}: \beta \in S_{\alpha}\right\}$ is $\mathcal{S}_{n}\left[\mathcal{A}_{2}\right]-$ admissible if $i>0$, and $\mathcal{S}_{n}$-admissible if $i=0$.

(4) For every node $f_{\alpha}$ in the levels $<\mathrm{h}(\mathcal{T})-i-1$, the set $\left\{f_{\beta}: \beta \in S_{\alpha}\right\}$ is $\mathcal{S}_{n}$ allowable.

Properties (2) and (3) give us also that every node in level $h(\mathcal{T})-i-1$ is also in the norming set of the space $Y$.

For $i=0$ properties (1) and (3) are consequences of (3.4), while property (2) follows from the definitions of the norming sets.

Assume that the inductive hypothesis holds for some $i \geq 0$. We shall transform the tree so that all the terminal nodes in the level $\mathrm{h}(\mathcal{T})-i-1$ become good terminal nodes. Let $f_{\alpha}= \pm e_{m_{\alpha}}^{*}$ be a bad terminal node in the level $\mathrm{h}(\mathcal{T})-i-1$, such that minsupp $f_{\alpha} \leq \operatorname{minsupp} f_{\gamma}$ for all the bad terminals nodes $f_{\gamma}$ in the level $\mathrm{h}(\mathcal{T})-i-1$.

Then we have that all the terminal nodes $f_{\beta}$ with $|\beta|=|\alpha|$ and minsupp $f_{\beta}<$ minsupp $f_{\alpha}$ are good. Also from the inductive hypothesis we have that for all the terminal nodes $f_{\beta}$, with $|\beta|>|\alpha|$ are good.

Let $f_{\beta}$ be the node on the level of $\alpha$ with largest minsupp $f_{\beta}<\operatorname{minsupp} f_{\alpha}$. Such a node exists otherwise there is nothing to the lower left of $f_{\alpha}$ and $f_{\alpha}$ would be a good terminal node. Also $S_{\beta} \neq \emptyset$, otherwise $f_{\beta}$ would be a good terminal node and anything to the lower left of $f_{\alpha}$ would also be to the lower left of $f_{\beta}$, which would imply that $f_{\alpha}$ is also a good terminal node.

Let $m_{\beta}=\operatorname{minsupp} f_{\beta}$. Since $\pm e_{m_{\beta}}^{*}$ is a good terminal node it follows that for every node $f_{\gamma}$ to the lower left of $f_{\beta}$ it holds that $\max \operatorname{supp} f_{\gamma}<\operatorname{minsupp} f_{\beta}$.

Indeed let $f_{\gamma}$ be a node to the lower left of $f_{\beta}$. If $|\gamma|=|\beta|$ and $f_{\gamma}$ is terminal node, it follows readily that maxsupp $f_{\gamma}=\operatorname{minsupp} f_{\gamma}<\operatorname{minsupp} f_{\beta}$. If $|\gamma| \geq|\beta|$ and $f_{\gamma}$ has successors or $|\gamma|>|\beta|$ and $f_{\gamma}$ is a terminal node, then from the inductive hypothesis we have that $\pm e_{\lambda_{\gamma}}^{*}$, where $\lambda_{\gamma}=\operatorname{maxsupp} f_{\gamma}$, is a good terminal node. Arguing as in Lemma 3.6, we get that maxsupp $f_{\gamma}<\operatorname{minsupp} f_{\beta}$.

From this observation and (3.4) we get that any node that causes $f_{\alpha}= \pm e_{m_{\alpha}}^{*}$ to be a bad terminal node is successor of $f_{\beta}$.

Let $E_{\beta}^{1}$ be the interval [minsupp $f_{\beta}, m_{\alpha}$ ) of $\mathbb{N}$ and $E_{\beta}^{2}$ be the interval $\left(m_{\alpha}\right.$, maxsupp $f_{\beta}$ ]. We set $f_{\beta}^{1}=E_{\beta}^{1} f_{\beta}$ and $f_{\beta}^{2}=E_{\beta}^{2} f_{\beta}$. By the inductive hypothesis $f_{\beta}$ belongs to the norming set of the space $Y$, and therefore $f_{\beta}^{1}, f_{\beta}^{2}$ belong also to the norming set of $Y$ (see comment after Definition 2.2). Since the intervals $E_{\beta}^{1}, E_{\beta}^{2}$ is a 
partition of the support of $f_{\beta}$, we have that $f_{\beta}=f_{\beta}^{1}+f_{\beta}^{2}$ and moreover by the choice of these intervals it holds that $f_{\beta}^{1}<f_{\alpha}=e_{m_{\alpha}}^{*}<f_{\beta}^{2}$. We replace the node $f_{\beta}$ and its successors by $f_{\beta}^{1}$ and the successors of $f_{\beta}^{1}$, i.e. we take the intersection of the successors of $f_{\beta}$ with the interval $E_{\beta}^{1}$.

Note that this replacement of $f_{\beta}$ by the node $f_{\beta}^{1}$ changes automatically the immediate predecessor $f_{\delta}$ of $f_{\beta}$ to $f_{\delta}^{1}$. It follows readily that minsupp $f_{\delta}=\operatorname{minsupp} f_{\delta}^{1}$ and hence this replacement changes neither the number nor the admissibility of the nodes of the previous levels.

We have now to add $f_{\beta}^{2}$ and its successors, which are the intersection of the successors of $f_{\beta}$ with the interval $E_{\beta}^{2}$, as nodes of the tree, and this in combination with the replacement of $f_{\beta}$ by $f_{\beta}^{1}$ will give us the new tree. Let

$$
\mathcal{A}=\left\{\gamma \in \mathcal{T}:|\gamma|=|\alpha| \text { and } \operatorname{minsupp} f_{\alpha} \leq \operatorname{minsupp} f_{\gamma}\right\}
$$

and enumerate the elements of $\mathcal{A}$ as $\alpha_{1}=\alpha, \alpha_{2}, \ldots$ so that the sequence (minsupp $f_{\alpha_{i}}$ ) $i_{i \geq 1}$ is increasing.

For the nodes in $\mathcal{A}$ which have successors the following key property holds:

If $\alpha_{i} \in \mathcal{A}$ and $f_{\alpha_{i}}$ has successors then maxsupp $f_{\beta}^{2}=\operatorname{maxsupp} f_{\beta}<\operatorname{minsupp} f_{\alpha_{i}}$

Indeed, let $\alpha_{i} \in \mathcal{A}$ be such that $f_{\alpha_{i}}$ has successors. Then if $m_{\alpha_{i}}=\operatorname{minsupp} f_{\alpha_{i}}$ it holds that $e_{m_{\alpha i}}^{*}$ is a good terminal node, since $\left|f_{\alpha}\right|<\left|e_{m_{\alpha i}}^{*}\right|$. Also $e_{\lambda_{\beta}}^{*}$, where $\lambda_{\beta}=\operatorname{maxsupp} f_{\beta}$, is a terminal node. Arguing as in the proof of Lemma 3.6 we get that either $e_{\lambda_{\beta}}^{*}$ is to the lower left of $e_{m_{\alpha i}}^{*}$ and hence $e_{\lambda_{\beta}}^{*}<e_{m_{\alpha i}}^{*}$ or $e_{\lambda_{\beta}}^{*}<\operatorname{minsupp} f_{\delta}$, where $f_{\delta}$ is the predecessor of $e_{m_{\alpha i}}^{*}$ in the level of $e_{\lambda_{\beta}}^{*}$. Thus in any case we have that $e_{\lambda_{\beta}}^{*}<e_{m_{\alpha i}}^{*}$ or equivalently $\operatorname{maxsupp} f_{\beta}^{2}=\operatorname{maxsupp} f_{\beta}<\operatorname{minsupp} f_{\alpha_{i}}$.

Let

$$
j=\max \left\{i: \operatorname{minsupp} f_{\alpha_{i}}<\operatorname{minsupp} f_{\beta}^{2}\right\} .
$$

From (3.5) it follows that $f_{\alpha_{i}}, i \leq j$, are terminal nodes. In order to preserve (3.4), $f_{\beta}^{2}$ must be added between $f_{\alpha_{j}}$ and $f_{\alpha_{j+1}}$.

We add $f_{\beta}^{2}$ to the immediate successors of the predecessor node $f_{\delta}$ of $f_{\alpha_{j}}$ and the successors of $f_{\beta}^{2}$ are the intersection of the successors of $f_{\beta}$ with the interval $E_{\beta}^{2}$.

Note that since we add $f_{\beta}^{2}$ after the terminal node $f_{\alpha_{j}}$, and as an immediate successor of the predecessor node $f_{\delta}$ of the terminal node, any such operation changes neither the number nor the admissibility of the nodes of the previous level. This gives us that property (4) of the inductive hypothesis holds. This completes the inductive step.

Let $\mathcal{T}_{1}$ be the resulting tree. Every terminal node in $\mathcal{T}$ is on the same level in $\mathcal{T}_{1}$. The condition (3.4) remains true in $\mathcal{T}_{1}$ by construction. We have to show now that any such transformation does not change a good terminal node in the levels $\geq h(\mathcal{T})-i$ into a bad terminal node.

Claim 2. If $f_{\gamma}$ is a good terminal node in $\mathcal{T}$ with $|\gamma|>|\alpha|$ or $|\gamma|=|\alpha|$ and minsupp $f_{\gamma}<\operatorname{minsupp} f_{\alpha}$, then $f_{\gamma}$ remains good terminal node in $\mathcal{T}_{1}$. 
Proof of Claim 2. Let $f_{\gamma}$ be a good terminal node in $\mathcal{T}$ with $|\gamma|>|\alpha|$ or $|\gamma|=$ $|\alpha|$ and minsupp $f_{\gamma}<\operatorname{minsupp} f_{\alpha}$. Let $f_{\beta} \in \mathcal{T}_{1}$ be a node which is to the lower left of $f_{\gamma}$. Then there is a node $f_{\delta}$ in $\mathcal{T}$ to the lower left of $f_{\gamma}$ such that either $f_{\beta}=$ $f_{\delta}$ or $f_{\beta}$ is the projection of $f_{\delta}$ onto $E_{\beta}^{1}$ or $E_{\beta}^{2}$. If $|\gamma|=\mid \alpha$ then it follows from the choice of $\alpha$ that maxsupp $f_{\delta}<\operatorname{minsupp} f_{\gamma}$, and if $|\gamma|>|\alpha|$ then it follows from the inductive hypothesis. Hence maxsupp $f_{\beta}<\operatorname{minsupp} f_{\gamma}$, and $f_{\gamma}$ is a good terminal node in $\mathcal{T}_{1}$.

We repeat the above steps, changing a bad terminal node into a good terminal one, for all the bad nodes in the level $\mathrm{h}(\mathcal{T})-i-1$. Since the nodes in the level $\mathrm{h}(\mathcal{T})-i-1$ is an $\mathcal{S}_{n(\mathrm{~h}(\mathcal{T})-i-1)}$ allowable set and hence finite, a finite number of such transformations will turn every bad terminal node into a good.

In the final step we check that the tree $\mathcal{T}_{1}$ satisfies properties (1)-(4) of the inductive hypothesis.

Claim 3. The tree $\mathcal{T}_{1}$ satisfies properties (1)-(3) of the inductive hypothesis, in every level $\geq h(\mathcal{T})-i-1$ and property (4) in every level $<h(\mathcal{T})-i-2$

Proof of Claim 3. Property (1) follows from Claim 2. Property (2) is a consequence of the inductive hypothesis and the properties of the norming set (see remark after Definition 2.2).

From the construction it follows that in the level $h(\mathcal{T})-i-1$ of $\mathcal{T}$ we add at most one node after each of its terminal nodes. Therefore after we complete the transformations in the level $\mathrm{h}(\mathcal{T})-i-1$, and $f_{\delta}$ is a node in the tree $\mathcal{T}$ in the level $\mathrm{h}(\mathcal{T})-i-2$, with $\mathcal{S}_{n}$-allowable immediate successors in the level $\mathrm{h}(\mathcal{T})-i-1$, then the immediate successors of (the new) $f_{\delta}$ in the new tree is at most $\mathcal{S}_{n}\left[\mathcal{A}_{2}\right]$-admissible set. The admissibility of the successors, that is they have successive support, follows from proof of Lemma 3.6. Therefore property (3) also holds. Also as we have already observe in the proof of the inductive step, any such transformation changes neither the number nor the admissibility of the nodes in the level $\mathrm{h}(\mathcal{T})-i-2$ and hence property (4) also holds.

A finite number of such transformations will give us a new tree $\mathcal{T}_{1}$ and a functional $\left(g_{\alpha}\right)_{\alpha \in \mathcal{T}_{1}}$ in the norming set of $Y$, which is admissible and satisfies (3.3). This completes the proof of Proposition 3.4.

Let us observe that the proof of Proposition 3.4 is independent of the number $\frac{1}{2^{n}}$, that is, it holds also for the spaces $T_{M}\left[\mathcal{S}_{n}, \theta\right]$ and $T\left[\mathcal{S}_{n}\left[\mathcal{A}_{2}\right], \theta\right]$ for every $0<\theta<1$. Since Proposition 3.2 is also independent of the number $\frac{1}{2^{n}}$, Remark 3.3, combining Propositions 3.2 and 3.4 we have the following theorem.

TheOREM 3.7. Let $n \in \mathbb{N}$ and $0<\theta<1$. Then the spaces $T_{M}\left[\mathcal{S}_{n}, \theta\right]$ and $T\left[\mathcal{S}_{n}, \theta\right]$ are 3-isomorphic.

As we have mentioned, the special case of Theorem 3.7 when $n=1$ has been proved in [3] and [4]. The isomorphic constant they provide is a function of $\theta$.

Let us now turn to the distortion problem. E. Odell and N. Tomczak-Jaegermann [10], have introduced for every $n \in \mathbb{N}$, the following equivalent norms on Tsirelson's space:

$$
\|x\|_{j}^{n}=\max \left\{\|x\|_{\infty}, \frac{1}{2^{j}} \sup \left\{\sum_{i \in F}\left\|E_{i} x\right\|_{n}:\left\{E_{i}: i \in F\right\} \text { is } \mathcal{S}_{j^{-}} \text {admissible }\right\}\right\}
$$


for $j=0,1, \ldots, n-1$. The main result of E. Odell and N. Tomczak-Jaegermann in [10], is that there exists a constant $K$ so that the norms $\|\cdot\|_{j}^{n}$ do not $K$ - distort any subspace of $T$. More precisely they have proved the following result.

TheOREM. (E. Odell and N. Tomczak-Jaegermann) There exists a constant $K$, so that for all $Y \prec T$ and $n \in \mathbb{N}$ there exist $Z \prec Y$ and $d>0$ such that for all $0 \leq j<n$ and $z \in S_{Z}$,

$$
d \leq\|z\|_{j}^{n} \leq K d
$$

Let us define the modified version, $\|\cdot\|_{j, n}^{M}$ of the previous norms:

$$
\|x\|_{j, M}^{n}=\max \left\{\|x\|_{\infty}, \frac{1}{2^{j}} \sup \left\{\sum_{i \in F}\left\|E_{i} x\right\|_{n}^{M}:\left\{E_{i}: i \in F\right\} \text { is } \mathcal{S}_{j} \text {-allowable }\right\}\right\}
$$

for $j=0,1, \ldots, n-1$. Combining the theorem of E. Odell and N. TomczakJaegermann and the 3-equivalence of the norms $\|\cdot\|_{n}$ and $\|\cdot\|_{n}^{M}, n \in \mathbb{N}$ we have the following theorem.

THEOREM 3.8. There exist a constant $K>1$ so that for all $Y \prec T$ and $n \in \mathbb{N}$ there exists $Z \prec Y$ and $d>0$ so that for every $z \in S_{Z}$,

$$
d \leq\|z\|_{j, M}^{n} \leq K d .
$$

\section{REFERENCES}

1. D. E. Alspach and S. A. Argyros, Complexity of weakly null sequences, Dissertationes Math 321 (1992), 1-44.

2. S. A. Argyros, I. Deliyanni, D. N. Kutzarova and A. Manoussakis, Modified mixed Tsirelson spaces, J. Funct. Anal. 159 (1998), 43-109.

3. S. Bellenot, Tsirelson superspaces and $\ell_{p}$, J. Funct. Anal. 69 (1986), 207-228.

4. P. G. Casazza and E. Odell, Tsirelson's space and minimal subspaces, Longhorn notes, (University of Texas 1982-3), 61-72.

5. P. G. Casazza and T. J. Shura, Tsirelson's space, Lecture Notes in Mathematics No. 1363 (Springer-Verlag, 1989).

6. W. B. Johnson, A reflexive Banach space which is not sufficiently Euclidean, Studia Math. 55 (1976), 201-205.

7. W. B.Johnson and J. Lindenstrauss, eds. Handbook of the geometry of Banach spaces Vol. 1 (Elsevier, Amsterdam, 2002).

8. W. B.Johnson and J. Lindenstrauss, eds. Handbook of the geometry of Banach spaces Vol. 2 (Elsevier, Amsterdam, 2003).

9. J. Lindenstrauss and L. Tzafriri, Classical Banach spaces Vol. I (Springer Verlag, 1977).

10. E. Odell and N. Tomczak-Jaegermann, On certain equivalent norms on Tsirelson's space, Illinois J. Math. 44 (2000), 51-71.

11. E. Odell, N. Tomczak-Jaegermann and R. Wagner, Proximity to $\ell_{1}$ and distortion in asymptotic $\ell_{1}$ spaces, J. Funct. Anal. 150 (1997), 101-145. 